University of Wollongong

Research Online

Faculty of Engineering - Papers (Archive)

Faculty of Engineering and Information

Sciences

December 2002

\title{
Terahertz-driven nonlinear electrical transport in semiconductor
} nanostructures

C. Zhang

University of Wollongong, czhang@uow.edu.au

Follow this and additional works at: https://ro.uow.edu.au/engpapers

Part of the Engineering Commons

https://ro.uow.edu.au/engpapers/39

\section{Recommended Citation}

Zhang, C.: Terahertz-driven nonlinear electrical transport in semiconductor nanostructures 2002.

https://ro.uow.edu.au/engpapers/39

Research Online is the open access institutional repository for the University of Wollongong. For further information contact the UOW Library: research-pubs@uow.edu.au 


\title{
TERAHERTZ-DRIVEN NONLINEAR ELECTRICAL TRANSPORT IN SEMICONDUCTOR NANOSTRUCTURES
}

\author{
C. Zhang \\ Department of Engineering Physics, University of Wollongong, \\ New South Wales 2522, Australia
}

\begin{abstract}
In this work, we used the quantum transport equation and density matrix formalism to calculate the frequency dependent electrical current of a two-dimensional electron gas directly driven by an intense terahertz laser. It is found that due to increased electron-photon coupling, the electron-impurity scattering decreases rapidly with the electric field.
\end{abstract}

\section{INTRODUCTION}

Terahertz $(\mathrm{THz})$ radiation has been applied to experimental investigation of nonlinear transport and optical properties in electron gases such as low dimensional semiconductor systems. Interesting new phenomena have been found in recent years, including resonant absorption[1], photon enhanced hot-electron effect[2,3], THz photon-induced impact ionization[4], LOphonon bottleneck effect[5], $\mathrm{THz}$ photon assisted tunneling[6], THz cyclotron resonance[7], $\mathrm{THz}$ switching effects in tunneling diode[8-10]. Despite the rapid development of terahertz phenomena, a theoretical formalism describing the quantum transport in strongly coupled electron-photon systems is lacking. In this paper, we present a theoretical investigation of the electrical current directly driven by the intense $\mathrm{THz}$ radiation field in the presence of electron-impurity scattering. We calculate the density matrix of the electron-photon system to the second order of impurity potential. The total current consists of contributions of successively electron-photon side bands. The strong nonlinear dependence of the real part of the current on the laser field indicates that as the electron-photon coupling becomes stronger, the electron-impurity scattering decreases rapidly.

\section{FORMALISM OF NONLINEAR ELECTRICAL CURRENT}

Our model system is a two dimensional electron gas under an intense laser radiation. We choose the laser field to be along the x-direction, $\mathbf{E}(t)=E_{0} \cos (\omega t) \mathbf{e}_{\mathbf{x}}$, where $E_{0}$ and $\omega$ are the amplitude and frequency of the laser field. For the notational convenience, both $\hbar$ and the speed of light $c$ have been set to unity. We shall also neglect the effect of the magnetic field component of the laser field since it is smaller than the electrical field component by the factor of $c$. Let us choose the vector potential for the laser field to be in the form $\mathbf{A}=\left(E_{0} / \omega\right) \sin (\omega t) \mathbf{e}_{\mathbf{x}}$. The time-dependent Schrödinger equation for a single electron is given as,

$$
i \frac{\partial}{\partial t} \psi(\mathbf{r}, t)=H \psi(\mathbf{r}, t)=\frac{(\mathbf{p}-e \mathbf{A})^{2}}{2 m^{*}} \psi(\mathbf{r}, t) .
$$

The time-dependent wavefunction can be written as,

$$
\psi_{\mathbf{k}}(\mathbf{r}, t)=\exp \left(-i 2 \gamma_{1} \omega t\right) \exp \left(i \gamma_{0} k_{x}(1-\cos (\omega t))\right) \exp \left(i \gamma_{1} \sin (2 \omega t)\right) \exp \left(-i \epsilon_{k} t\right) \exp (i \mathbf{k} \cdot \mathbf{r})
$$


where $\gamma_{0}=\left(e E_{0}\right) / m^{*} \omega^{2}$ and $\gamma_{1}=\left(e E_{0}\right)^{2} /\left(8 m^{*} \omega^{3}\right)$. The effect of electrical field is included in this wavefunction exactly. These wavefunctions satisfy the orthonormal condition and can be used as the basis for constructing the quantum field operator,

$$
\Psi(\mathbf{r}, t)=\sum_{\mathbf{k}} a_{\mathbf{k}} \psi_{\mathbf{k}}(\mathbf{r}, t), \quad \Psi^{\dagger}(\mathbf{r}, t)=\sum_{\mathbf{k}} a_{\mathbf{k}}^{\dagger} \psi_{\mathbf{k}}^{*}(\mathbf{r}, t)
$$

where $a_{\mathbf{k}}^{\dagger}\left(a_{\mathbf{k}}\right)$ is the creation (annihilation) operator for the electronic state with momentum k. These field operators satisfy the equal time commutation relation, $\left\{\Psi^{\dagger}(\mathbf{r}, t), \Psi\left(\mathbf{r}^{\prime}, t\right)\right\}=$ $\delta\left(\mathbf{r}-\mathbf{r}^{\prime}\right)$. The field operators can also be written in terms of eigenfunctions of a free electron,

$$
\Psi(\mathbf{r}, t)=\sum_{\mathbf{k}} b_{\mathbf{k}}(t) e^{i \mathbf{k} \cdot \mathbf{r}}, \quad \Psi^{\dagger}(\mathbf{r}, t)=\sum_{\mathbf{k}} b_{\mathbf{k}}^{\dagger}(t) e^{-i \mathbf{k} \cdot \mathbf{r}},
$$

where $b_{\mathbf{k}}(t)=a_{\mathbf{k}} \exp \left(-i 2 \gamma_{1} \omega t\right) \exp \left(i \gamma_{0} k_{x}(1-\cos (\omega t))\right) \exp \left(i \gamma_{1} \sin (2 \omega t)\right)$.

We now calculate the electrical current of system driven by the terahertz laser due to electron-random-impurity scattering. The Hamiltonian of the system, in the second quantized notation, can be written as, $H=H_{0}+H_{e e}+H_{e I}$. Here $H_{0}$ is the Hamiltonian of a noninteracting many-electron system,

$$
H_{0}=\frac{1}{2 m^{*}} \sum_{\mathbf{p}}[\mathbf{p}+e \mathbf{A}]^{2} b_{\mathbf{p}}^{\dagger}(t) b_{\mathbf{p}}(t)
$$

$H_{e e}$ is the electron-electron interaction[11],

$$
H_{e e}=\frac{1}{2} \sum_{\mathbf{p}, \mathbf{p}^{\prime}, \mathbf{q}} V_{q} b_{\mathbf{p}+\mathbf{q}}^{\dagger}(t) b_{\mathbf{p}^{\prime}-\mathbf{q}}^{\dagger}(t) b_{\mathbf{p}^{\prime}}(t) b_{\mathbf{p}}(t)=\frac{1}{2} \sum_{\mathbf{p}, \mathbf{p}^{\prime}, \mathbf{q}} V_{q} a_{\mathbf{p}+\mathbf{q}}^{\dagger}(t) a_{\mathbf{p}^{\prime}-\mathbf{q}}^{\dagger}(t) a_{\mathbf{p}^{\prime}}(t) a_{\mathbf{p}}(t),
$$

where $V_{q}=2 \pi e^{2} / q$ is the Fourier transform of electron-electron interaction in two dimensions. $H_{e I}$ is the interaction between the electrons and random impurities,

$$
H_{e I}=-\sum_{\mathbf{p}, \mathbf{q}} V_{q} b_{\mathbf{p}+\mathbf{q}}^{\dagger}(t) b_{\mathbf{p}}(t) \sum_{i} e^{i \mathbf{q} \cdot \mathbf{R}_{\mathbf{i}}}
$$

where we assumed that impurities are singly charged. $\mathbf{R}_{\mathbf{i}}$ is the position of $i^{\text {th }}$ impurity. The total average $2 \mathrm{D}$ current density of the system is defined as,

$$
\mathbf{j}=\left\langle\frac{\delta H}{\delta \mathbf{A}}\right\rangle=\frac{e}{m^{*}} \sum_{\mathbf{p}}\left\langle[\mathbf{p}+e \mathbf{A}] b_{\mathbf{p}}^{\dagger}(t) b_{\mathbf{p}}(t)\right\rangle=\mathbf{j}_{1}+\mathbf{j}_{0}
$$

Since $\mathbf{A}=(E / \omega) \sin (\omega t) \mathbf{e}_{\mathbf{x}}$,

$$
\mathbf{j}_{0}=\frac{e^{2}}{m^{*}} e \mathbf{A} \sum_{\mathbf{p}}\left\langle b_{\mathbf{p}}^{\dagger}(t) b_{\mathbf{p}}(t)\right\rangle=\frac{e^{2} n}{m \omega} \sin (\omega t) E_{0} \mathbf{e}_{\mathbf{x}},
$$

where $n$ is the $2 \mathrm{D}$ electron concentration. The electrical field is oscillating as $\cos (\omega t)$. Therefore $\mathbf{j}_{0}$ is in the direction of the laser field but its phase is behind the phase of the electrical field by $\pi$. All effects on the electrical current due to the laser field is contained in the current density

$$
\mathbf{j}_{1}=\frac{e}{m^{*}} \sum_{\mathbf{p}}\left\langle\mathbf{p} b_{\mathbf{p}}^{\dagger}(t) b_{\mathbf{p}}(t)\right\rangle
$$


We will use the density matrix method to evaluate $\mathbf{j}_{1}$. The equation of motion for the singleelectron density matrix $F(\mathbf{p}, \mathbf{p}+\mathbf{k})=\left\langle b_{\mathbf{p}}^{\dagger}(t) b_{\mathbf{p}+\mathbf{k}}(t)\right\rangle$ is given as

$$
\begin{gathered}
i \frac{\partial}{\partial t} F(\mathbf{p}, \mathbf{p}+\mathbf{k})=\left[\epsilon_{\mathbf{p}+\mathbf{k}}-\epsilon_{\mathbf{p}}+k_{x} \gamma_{0} \omega \sin (\omega t)\right] F(\mathbf{p}, \mathbf{p}+\mathbf{k}) \\
+\sum_{\mathbf{q}} V_{q}\left[n(\mathbf{q}, t)-\sum_{i} e^{i \mathbf{q} \cdot \mathbf{R}_{\mathbf{i}}}\right][F(\mathbf{p}, \mathbf{p}+\mathbf{k}-\mathbf{q})-F(\mathbf{p}+\mathbf{q}, \mathbf{p}+\mathbf{k})] .
\end{gathered}
$$

Here $\epsilon_{\mathbf{p}}=p^{2} / 2 m^{*}$ is the kinetic energy of an electron having momentum $\mathbf{p}$, and $n(\mathbf{q}, t)=$ $\sum_{\mathbf{p}} F(\mathbf{p}, \mathbf{p}+\dot{\mathbf{q}})$. The time-dependent current density $\mathbf{j}_{1}(t)$, is now given as

$$
i \frac{d \mathbf{j}_{1}(t)}{d t}=\frac{-e}{m^{*}} \sum_{\mathbf{q}} V_{q} \mathbf{q} n(-\mathbf{q}, t) \sum_{i} e^{i \mathbf{q} \cdot \mathbf{R}_{\mathbf{i}}}
$$

We have recently developed a method to calculate the density matrix in a strongly coupled electron-photon system[11]. Making use of the generating function for the Bessel function, $\exp [i \alpha \cos (x)]=\sum_{m} i^{m} J_{m}(\alpha) \exp (i m x)$ (where $J_{m}$ is the Bessel function of first kind), the density matrix, the density fluctuation, the total current can be decomposed into a sum of successive harmonics. We solve for the electron density matrix up to the second order in electron-impurity interaction, $F_{1}$. The solution of the m-th order density matrix, $F_{1}^{(m)}$ is given as

$$
F_{1}^{(m)}=-\frac{V_{k}}{2 \pi D(k, m \omega)} \frac{f_{\mathbf{p}+\mathbf{k}}-f_{\mathbf{p}}}{\epsilon_{\mathbf{p}+\mathbf{k}}-\epsilon_{\mathbf{p}}-m \omega} \sum_{i} e^{i \mathbf{k} \cdot \mathbf{R}_{\mathbf{i}}}
$$

where $D(q, m \omega)=1-V_{q} Q(q, m \omega)$ is the dielectric function in the random-phase-approximation and $Q(q, \omega)$ is the the polarizability for free electrons. The final result for the electric current can be written as

$$
\mathbf{j}_{1}(\omega)=\frac{-n e}{m^{*} \omega} \sum_{\mathbf{q}} \mathbf{q} V_{q} \sum_{m} \frac{(-i)^{m}}{m} J_{m}\left(q_{x} \gamma_{0}\right) e^{i q_{x} \gamma_{0}} \frac{V_{q} Q(q, m \omega)}{D(q, m \omega)} .
$$

For isotropic systems, the electric current is along the direction of polarization of the laser field. The real part of the electric current is given as

$$
\begin{gathered}
\Re e\left[\mathbf{j}_{1 x}(\omega)\right]=-\frac{n e}{m^{*} \omega} \sum_{\mathbf{q}} q_{x} V_{q} \sum_{m} \frac{J_{m}\left(q_{x} \gamma_{0}\right)}{m} \times \\
\left\{\Im m\left[\frac{1}{D(q, m \omega)}\right] \sin \left(q_{x} \gamma_{0}-m \pi / 2\right)-\Re e\left[\frac{1}{D(q, m \omega)}\right] \cos \left(q_{x} \gamma_{0}-m \pi / 2\right)\right\} .
\end{gathered}
$$

We now make use of the following facts: (a) The dielectric function is only dependent on the magnitude of $q$; (b) The Bessel functions are symmetric for even $m$, and antisymmetric for odd $m$ with respect to the argument $q_{x}$. Therefore the integration over the direction of $q$ will be nonzero for the second term in the curl brackets. The real part of the electric current is now written as,

$$
\Re e\left[\mathbf{j}_{1 x}(\omega)\right]=-\frac{n e}{m^{*} \omega} \sum_{\mathbf{q}} q_{x} V_{q} \sum_{m} \frac{J_{m}\left(q_{x} \gamma_{0}\right)}{m} \Im m\left[\frac{1}{D\left(q_{.} m \omega\right)}\right] \sin \left(q_{x} \gamma_{0}-m \pi / 2\right) .
$$




\section{RESULTS AND DISCUSSIONS}

We have numerically calculated real part of the current. The parameters used in our calculation are those of GaAs, $m^{*}=0.067 m_{0}, r_{s}=m^{*} e^{2} /\left(\hbar^{2} k_{F}\right)=1.0 . R=k_{F} e E_{0} /\left(m^{*} \omega^{2}\right)$ is the reduced electric field.

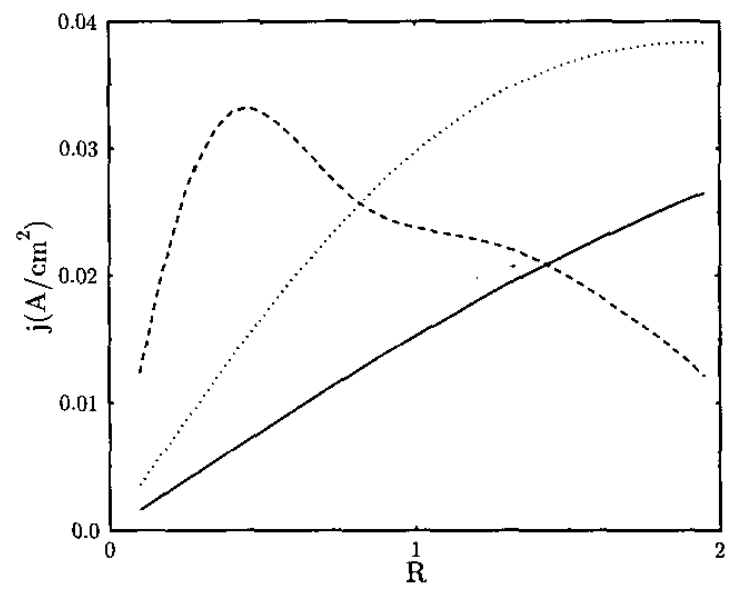

Fig.1. Electrical current as a function of the reduced electric field $\mathrm{R}$ for GaAsbased semiconductor quantum wells. The solid line is for $\omega=6 \mathrm{THz}$, the dotted line is for $\omega=4 \mathrm{THz}$, and the broken line is for $\omega=2 \mathrm{THz}$.

Fig.1 shows electric field dependence of the real part of the electric current. The real part of the current is a direct measure of the absorption of $\mathrm{THz}$ photons by the electronic system. At weak electric fields or high frequencies, the current is almost linear in the electric field. The electron-photon coupling is inversely proportional to $\omega^{2}$. Therefore the absorption increases as frequency decreases. At fixed frequency, as the laser intensity increases, the current starts to deviate from the linear dependence, the absorption coefficient $j / E$ starts to decrease with the field intensity. This behaviour is a direct consequence of the electron-impurity scattering time being affected by electron-photon coupling. At low field intensity or weak electronphoton coupling, the scattering time is independent of the electric field. As field intensity increases, electrons-photon coupling become stronger and as a consequence, the electronimpurity scattering becomes less effective. This reduced electron-impurity scattering is the origin of the reduction of the current at high fields.

\section{REFERENCES}

[1] N. G. Asmar, et al., Phys. Rev. B51, 18041 (1995)

[2] N. G. Asmar, et al, Appl. Phys. Lett. 68, 829 (1996)

[3] W. Xu and C. Zhang, Phys. Rev. B55, 5259 (1997); Appl. Phys. Lett. 68, 3305 (1996)

[4] A. G. Markelz, N. G. Asmar, B. Brar, and E. G. Gwin, Appl. Phys. Lett. 69, 3975 (1996)

[5] B. N. Murdin,et al., Phys. Rev. B55 5171 (1997)

[6] C. J. G. M. Langerak, et al., Appl. Phys. Lett. 67, 3453 (1995)

[7] T. A. Vaughan,et al., Phys. Rev. B53 16481 (1996)

[8] C. Zhang, Appl. Phys. Lett. 78, 4187 (2001)

[9] P. Orellana, F. Claro, and E. Anda, Phys. Rev. B62, 9959 (2000)

[10] P. Orellana and F. Claro, Appl. Phys. Lett. 75, 1643 (1999)

[11] C. Zhang, Phys. Rev. B 66, 081105(R) (2002) 